\title{
Constructing High Resolution Satellite Image Using Interpolation Algorithm And Fast Nlm Filter
}

\author{
S.Sudaroli ${ }^{1}$, ChitraValavan ${ }^{2}$ \\ PG Student, ECE Depatment, AVC college of engineering, Mayiladuthurai, India ${ }^{1}$ \\ Professor \& HOD,ECE Depatment, AVC college of engineering, Mayiladuthurai, India ${ }^{2}$
}

\begin{abstract}
In remote sensing, resolution of an image is limited by diffraction, atmospheric distortion and imperfect focus. Our proposed Resolution Enhancement(RE) scheme uses Dual tree-complex wavelet transform(DT-CWT), Lanczos interpolation and Fast NLM filtering for post processing. Fast Non Local Means filtering speed up the processing time compared to the NLM filtering. The Lanczos interpolator is used to interpolate the high frequency subbands and low resolution input image. Now these high frequency sub-bands are passed through Fast NLM filter for good clarity. Inverse DT-CWT is applied to obtain resolution enhanced image. In parallel performance of Fast NLM filter is also calculated for different set of input images using Mean Square Error(MSE) and Signal-to-noise ratio(SNR).
\end{abstract}

Key Words: Lanczos interpolator, Fast NLM filter, Mean Square Error(MSE) and Signal-to-noise ratio(SNR), Summed Square Index (SSI), Dual tree-complex wavelet transform(DT-CWT).

\section{INTRODUCTION}

Resolution is the major limitation for using the satellite images in many applications like monitoring remote areas, geo science studies, geographical information systems and astrology. Clarity of the image is decided by its resolution. Pixel size is determined by the sampling distance, so to enhance the resolution in image we have to increase the number of pixels which means inserting more sampling points. Transformations are applied to images to obtain further information from the image that is not readily available in the raw signal. The high frequency and low frequency components of an image is needed to enhance the image without the loss of any information from the given image[1]. Our transformed image will contain many sub bands which provide detailed informations about high frequency and low frequency components.

\section{PRELIMINARIES}

a) DTCWT has two filter banks. It employs two DWT, first DWT gives the real part and second DWT gives the imaginary part.It keeps the real and imaginary parts of the complex coefficient separately.[2]In DTCWT, a real signal is applied to the both trees for decomposition and the outputs of the both reconstructed trees are added at the end of the reconstruction stage.

b) Interpolator tries to achieve a best approximation of a pixel's intensity based on the values at surrounding pixels but it can never add detail to the image which is not already present. The intensity values of pixels are interpolated along the $x$-axis to produce four intermediate results. Then the intensity is computed by interpolating the intermediate values along the $y$ axis.[10]Lanczos Interpolator reconstructs the signal lost in the sampling process by smoothing the data samples with sinc interpolation function.

c) Fast non local means filter which is improvements to non-local means (NLM) image denoising method is used here to reduce the computational complexity. In the basic NLM algorithm, neighbourhood pixel weightages are computed using the window similarity method.

This Fast NLM also uses the concept of filtering out the non-similar neighborhood pixels which based on fixed sized window gray mean values.

Also the mean values of variable sized windows in image are computed efficiently using summed image (SI) concept, this requires only 3 additions. The Fast NLM filter performance is nearly 80 times faster than NLM algorithm.

d) Inverse DT CWT is used in the synthesis part of the tree hence it reconstructs the decomposed subband coefficiets.It performs the down sampling.

\section{PROPOSED SYSTEM}

Interpolation is one of the simple and efficient method for increasing the number of pixels in image.It is the process of estimating intermediate values of a sampled functions at continuous position.

There are different types of interpolators available in image processing (Nearest neighbour, bilinear, cubic, Lanczos).Among these Lanczos interpolator gives the best result. 
The Lanczos interpolation belongs to the method of "windowed sinc" function.

$\mathrm{L}(\mathrm{x})=\sin (\mathrm{x}) \cdot \operatorname{Lanc} 2(\mathrm{x})=\left\{\begin{array}{cl}\sin (\pi x) \frac{n}{\pi x} & , 0 \leq|x|<2(1) \\ 0 & \end{array}\right.$

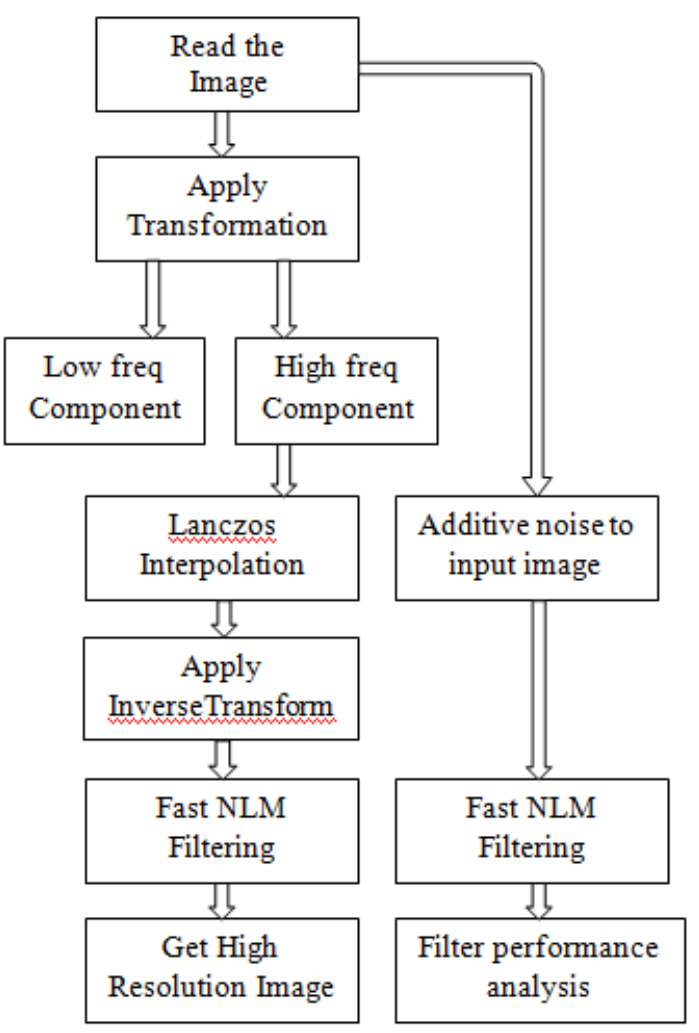

Fig. 1.Block Diagram

Images are often corrupted by noise during acquisition, so that effective noise suppression methods are required. The Non-local means (NL-means) is a popular technique developed by Buades et al[16]. Its efficiency and conceptual simplicity have made it very popular, but its main drawback is its high computational complexity. For an image of size $\mathrm{N} \times \mathrm{M}$, patches of size $7 \times 7$ and a search window of size $21 \times 21$, which is a typical use setting, the complexity is 72.212.N.M. This makes accelerations necessary to maintain low computation times. Numerous methods have been proposed to accelerate the NL-means, such as preselection of the contributing neighborhoods based on average value and gradient, average and variance, higher-order statistical moments, cluster tree arrangement, mean values at different resolutions, or probabilistic early termination

\section{FAST NLM FILTER}

Fast non-local means algorithm is based on Summed Squared Image (SSI) and fast Fourier transform (FFT), together with an approach for estimating the standard deviation of noise. An improvement over the computational speed of existing non local means filter give Fast NLM in which image quality towards existing algorithm is to aviod the contributions from the pixels in dissimilar windows during denoising. Although weights of dissimilar windows are seem to very small at first glance, the new estimated pixel value in image can be severely biased due to the many small contributions of dissimilar windows.

Now use a pre-classification technique, in which only weights for the non repeating most meaningful pixels are computed. This approach is a fast way to not include the dissimilar windows, which finally results in a lesser computational time and even in a better overall improvement in denoising. Our Fast non-local means algorithm is also optimized with the advantage of the symmetry in the weights to speed up the weight computations. For the convenience of computation, fast non-local means algorithm adopted the Euclidean distance to compare two neighbourhoods

$$
\begin{gathered}
\mathrm{S}(\mathrm{i} . \mathrm{j})=\|\mathrm{Ni}-\mathrm{Nj}\|^{2} \\
=\left[\sum_{l=0}^{M-1} \sum_{m=0}^{M-1}[\operatorname{Ii}(l, m)-\operatorname{Ij}(l, m)]^{2}\right]
\end{gathered}
$$

The principle of SSI resembles the Integral image which has been used in the application of face detection . For each pixel in the image, the integral image will maintains the summed value of all the existing pixels in the upper left part of the given image. Like the definition of integral image, for each pixel it stores the summed square values in the upper left pixels.SSI can also be

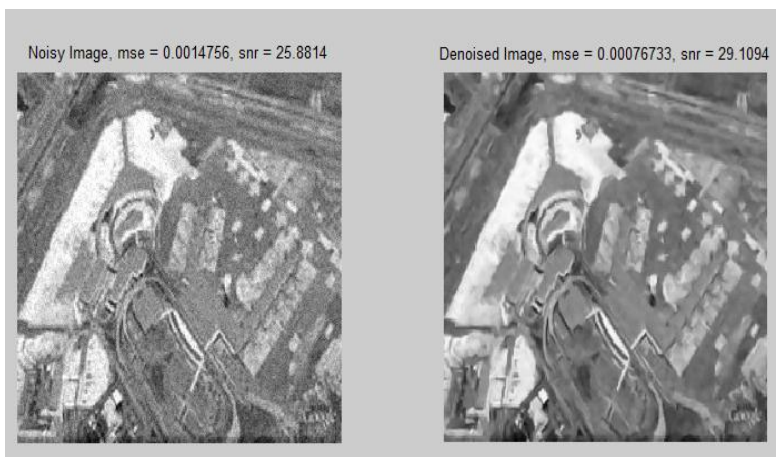

Fig. 2. Fast NLM filter performance analysis

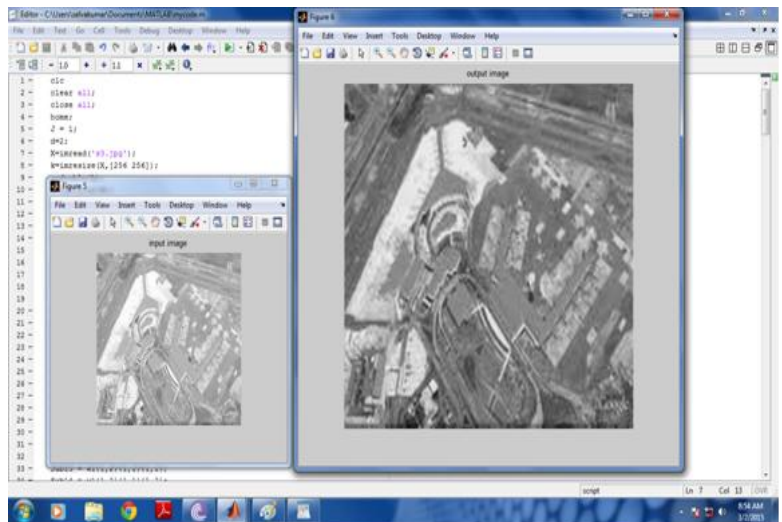

Fig. 3.High resolution output image

obtained interms of linear time proportional to the size of image, each pixel in the original image is processed only once, so the computational complexity for computing SSI is $O\left(\mathrm{x}^{2}\right)$, in which $\mathrm{x}$ is the size of the image. By using the SSI method, sum of squares can be easily obtained for 
each pixel in any rectangles of the image within constant time.

The Equation 2. denotes the caculation of Euclidean distance in the fast non local means filtering, $\mathrm{Ni}, \mathrm{Nj}$ denotes the neighbourhood pixels of two windows $\mathrm{i}, \mathrm{j}$ respectively. Ii,Ij in the Equation 3. indicates the two dissimilar windows in the integral image.

- Gaussian noise is added to the input image.

- Gaussian noise are random noise which are uniformly distributed functions.

- They occurred in image due to poor illumination.

- Then filtered it through Fast NLM filter.

- Mean squared error(MSE) and Signal-to-noise ratio(SNR) are calculated to analyse filter performance.

- The peak signal to noise ratio (PSNR) is commonly used to measure the image quality.

- $\quad \mathrm{PSNR}=10 \log 10 \frac{255 N^{2}}{\sum_{i, j=1}^{N}\left(x_{i, j}^{\prime}-x_{i, j}\right)^{2}}$

- $\mathrm{NxN}$ - pixels in image

- $x_{i, j}^{\prime}$-- reconstructed image

- $x_{i, j}$-- original image

- $0.5 \mathrm{~dB}$ PSNR improvement is considered to be visible.

\section{RESULT}

To improve the image quality Lanczos interpolator is used for the enhancement of resolution, the image sub bands are processed separately. The main disadvantage of using the non local means filter for post processing is its computational complexity, since it takes longer time to estimate the euclidean distance between the dissimilar windows.our proposed Fast nlm filter implemented Summed Squared Index(SSI), which will automatically calculate the Euclidean distance and also reduces the computational complexity of denoising process.

\section{CONCLUSION}

The clarity of the image is improved efficiently using Lanczos interpolator and also the complexity of denoising is limited with the use of fast non local means filter instead of non local means filter. Hence our proposed Fast nlm performs 80 times faster than nlm filter.Fig. 2 shows the performance of Fast NLM filter and Fig.3 shows our resolution enhanced image. We can also increase the number of pixels in the image it will results in some what better clarity but it may tend to computational complexity. In our high resolution image due to increase in number of pixels the object are seen clearly.

\section{REFERENCES}

[1] [Online].Available:http://www.satimagingcorp.com/

[2] Y. Piao, I. Shin, and H. W. Park, "Image resolution enhancement using inter-subband correlation in wavelet domain," in Proc. Int. Conf. ImageProcess., San Antonio, TX, 2007, pp. I-445-I-448.

[3] C. B. Atkins, C. A. Bouman, and J. P. Allebach, "Optimal image scaling using pixel classification," in Proc. Int. Conf. Image Process., Oct. 7-10, 2001, pp. 864-867.
[4] A. S. Glassner, K. Turkowski, and S. Gabriel, "Filters for commonresampling tasks," in Graphics Gems. New York: Academic, 1990,pp. 147-165.

[5] D. Tschumperle and R. Deriche, "Vector-valued image regularization with PDE's: A common framework for different applications," IEEE Trans.Pattern Anal. Mach. Intell., vol. 27, no. 4, pp. 506-517, Apr. 2005.

[6] M. J. Fadili, J. Starck, and F. Murtagh, "Inpainting and zooming using sparse representations," Comput. J., vol. 52, no. 1, pp. 64-79, Jan. 2009.

[7] H. Demirel and G. Anbarjafari, "Discrete wavelet transform-based satellite image resolution enhancement," IEEE Trans. Geosci. Remote Sens., vol. 49, no. 6, pp. 1997-2004, Jun. 2011.

[8] H. Demirel and G. Anbarjafari, "Image resolution enhancement by using discrete and stationary wavelet decomposition," IEEE Trans. Image Process., vol. 20, no. 5, pp. 1458-1460, May 2011.

[9] H. Demirel and G. Anbarjafari, "Satellite image resolution enhancement using complex wavelet transform," IEEE Geosci. Remote Sens. Lett., vol. 7, no. 1, pp. 123-126, Jan. 2010.

[10] H. Demirel and G. Anbarjafari, "Image super resolution based on interpolation of wavelet domain high frequency subbands and the spatial domain input image," ETRI J., vol. 32, no. 3, pp. 390-394, Jan. 2010

[11] H. Zheng, A. Bouzerdoum, and S. L. Phung, "Wavelet based nonlocalmeans super-resolution for video sequences," in Proc. IEEE 17th Int.Conf. Image Process., Hong Kong, Sep. 26-29, 2010, pp. $2817-2820$

[12] A. Gambardella andM.Migliaccio, "On the superresolution of microwave scanning radiometer measurements," IEEE Geosci. Remote Sens. Lett., vol. 5, no. 4, pp. 796-800, Oct. 2008. 